\title{
Antibiotic Therapy Associated Cardiotoxicity
}

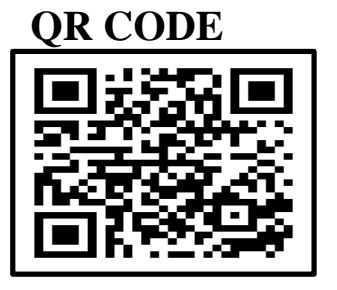

\section{MANISHA JHA ${ }^{1}$, AKRAM KHAN*2 $^{*}$}

The improper and frequent use of antibiotics has been on a rise. Empirical use for unapproved indications leads to development of resistant pathogens. Many other adverse drug reactions are associated with the use of antibiotics. These may be due to the direct effect of these drugs or due to their interactions with other therapeutic agents. Cardiac toxicities like QT prolongation, aortic aneurysms and dissections are of high concern and may result in long-term health risks. To prevent these adverse reactions, antibiotics should be used as per the manufacturer's and physician's instructions. The use of interacting drugs should be avoided wherever possible. Polypharmacy practice should be done after considering all the patient's health aspects. This review summarizes the cardiac toxicities of commonly prescribed antibiotics and their interactions with other drugs.

KEYWORDS: Antibiotics, Cardiac Toxicity, Adverse Reactions

\section{INTRODUCTION}

Medical literature suggests that antibiotics are frequently not appropriately prescribed and their use in everyday clinical practice is not based on an evidence centered approach. ${ }^{1,2}$ For instance, antibiotics are prescribed for viral fever and common cold, against which they are not effective. The chronic and inappropriate use of antibiotics leads to development of resistance. This development of bacterial resistance to antibiotics has been one of the most important challenges faced by medical professionals globally. Rational antibiotic therapy and patient information are important building blocks for maintaining the efficacy of these drugs, which are essential to modern medicine. It is however also important to consider other risks associated with antibiotic use. These include possible cardiovascular side effects and risks of antibiotic therapy as well as the risks resulting arising therefrom. Long-term use of antibiotics in middle-aged or older women may increase the risk of subsequent cardiovascular diseases. ${ }^{3}$ Possible causes include the following cardiac side effects or risks of antibiotics QT interval prolongation, risk of aortic aneurysms and dissection, adverse reactions due to clinically significant interactions with antibiotics.

Prolongation of the QTc interval is probably the most therapeutically significant and best-documented side effect of therapy with certain classes of antibiotics, which may lead to life-threatening polymorphic ventricular tachyarrhythmias, so-called torsades de pointes. ${ }^{4}$ Both fluoroquinolones and macrolides are associated with these side effects. Fluoroquinolones in general, and moxifloxacin in particular, have a direct effect on a specific potassium current which delays cardiac repolarization, reflected in a surface ECG as prolonged QT interval. This type of change in repolarization may result in the development of torsades de pointes in patients. Fluoroquinolones are no longer the first choice in the treatment of infections due to medically significant side effects. They should only be used if other and better tolerated antibiotics are unavailable. Severe musculoskeletal side effects include tendinitis, tendon rupture, myalgia, muscle weakness, arthralgia, joint swelling and gait disorders. Severe side effects involving the peripheral and central nervous systems include peripheral neuropathy, sleeplessness, depression, fatigue, impaired memory as well as visual, auditory, olfactory and gustatory disorders. Special caution is generally advised in elderly patients, those with renal problems or following organ transplantation, as well as patients being concomitantly administered systemic corticosteroids. Macrolides are widely used class of antibiotics used in the treatment of many common infections.

They are considered safe and are well tolerated in general. However, several studies have demonstrated an association between macrolides and cardiotoxicity 
in recent years. ${ }^{1,2}$ Reported adverse cardiac events due to macrolides include QT interval prolongation, torsades de pointes, ventricular tachycardia, and sudden cardiac death. These have especially been observed in patients with pre-existing cardiovascular diseases. All macrolides in current use carry a risk of QTc interval prolongation and/or torsades de pointes. A few studies have demonstrated an association between administration of macrolide antibiotics and sudden cardiac death. The extent of difference of this risk between the various macrolides remains controversial. Thus, certain precautions and attention to risk factors are recommended for all active substances. These include cardiovascular diseases, long QT syndrome, electrolyte disturbances and pharmacokinetic interactions..$^{5-16}$

It is important to take these risk factors into account and monitor patients accordingly or avoid the use of QT prolonging drugs in such cases whenever possible. In principle, the combination of the two antibiotic groups with other drugs which may also induce significant QT prolongation and torsades de pointes is critical. This combination is recommended only under intensive observation or is contraindicated. In principle, the combination of two or more drugs which may potentially prolong the QTc interval or increase the risk of TdP should be avoided. ${ }^{4}$ Thus, in polypharmacy, the possibility of using a QT-neutral drug with the same therapeutic efficacy instead of a QT-prolonging drug should be evaluated in every patient and must be given preference. If combination therapy is unavoidable, then the lowest effective dose must be selected and administered under ECG monitoring. Fluoroquinolones may promote the degradation of collagen. Predisposing factors for aortic aneurysms and dissection include: family history of aneurysm, pre-existing aortic aneurysm or aortic dissection, Marfan syndrome, vascular EhlersDanlos syndrome, Takayasu arteritis, giant cell arteritis, Behçet's disease, hypertension and atherosclerosis. Clarithromycin and erythromycin are potent $\mathrm{CYP}_{3} \mathrm{~A}_{4}$ and P-glycoprotein inhibitors. Caution is advised in combination with drugs which are primarily metabolized by $\mathrm{CYP}_{3} \mathrm{~A}_{4}$ or are good substrates for the transport protein P-gp due to the greater toxicity of these drugs. Concomitant administration of clarithromycin and calcium antagonists such as amlodipine and felodipine may lead to hypotension and acute kidney failure, with high mortality. Azithromycin is the drug of choice in such cases since it does not exhibit such pharmacokinetic interactions. The use of azithromycin is therefore recommended in polypharmacy. ${ }^{17}$

Cotrimoxazole is an important and potential trigger of hyperkalemia in elderly patients as well as in those with renal dysfunction, which is of particular significance during concomitant intake of ACE inhibitors or AT1 blockers. The trimethoprim component acts like the potassium-sparing diuretic amiloride. High cardiovascular mortality has been observed in combination with ACE inhibitors and angiotensin blockers. ${ }^{18}$ Changes in microbiomes and possible cardiovascular effects. The intestinal flora plays a key role in many physiological processes and pathological diseases. Every dose of the antibiotic affects the balance and composition of intestinal flora by alteration in the microbiome such as a possible reduction in the occurrence of probiotic bacteria. Findings from animal experiments suggest that exposure to antibiotics may also increase cardiovascular risk by alteration of the microbiome. Thus, intestinal bacteria, which enter serum via their metabolites and exhibit a protective effect against arteriosclerosis, may be reduced. Some antibiotics stimulate the proliferation and activity of macrophages in vitro, which may lead to accumulation of lipids and result in atherosclerosis in the long term. Recently conducted studies indicates an unexpected effect of antibiotics in furthering inflammation..$^{19-25}$

According to current recommendations, caution is advised while administering fluoroquinolones due to their side effects. These include cardiac toxicities and associated risks. The patient's individual risk factors must be considered while administering macrolides and concomitant use with other QT-prolonging drugs must be avoided at any cost. The macrolides clarithromycin and erythromycin exhibit drug interactions and should be avoided whenever possible. Azithromycin is the macrolide of choice in polypharmacy. In general, effects on the microbiome must be considered while prescribing antibiotics. This may also result in cardiac toxicities or long-term risks according to initial reports. It is therefore imperative that antibiotics be administered in accordance with the given indication, with therapy targeted at the treatment of bacterial infections. Restricted use of antibiotics is therefore important since it contributes 
not only to the reduction of bacterial resistance, but also towards reduction of cardiovascular risks.

\section{CONCLUSION}

The frequent use of antibiotics for inappropriate indications leads to selection of resistant pathogens, promotion of their spread and development of resistance. Other risks associated with the use of antibiotics must be considered. These include possible cardiovascular side effects of antibiotic therapy as well as the risks resulting arising therefrom, and include prolongation of the QTc interval, the risk of aortic aneurysms and dissection, side effects due to clinically significant interactions and changes in microbiomes with resultant possible side effects and risks. According to the current recommendations, caution is advised while administering fluoroquinolones due to their side effects. The patient's individual risk factors must be considered while administering macrolides and concomitant use with other QT-prolonging drugs must be avoided at any cost. Use of the macrolides clarithromycin and erythromycin which exhibit potent interactions should be avoided whenever possible. Azithromycin is the macrolide of choice in polypharmacy. In general, the effects on the microbiome must be considered when prescribing antibiotics. These may result in long-term cardiovascular risks based on initial reports. It is therefore imperative that antibiotics be administered in accordance with the given indication, with therapy targeted at the treatment of bacterial infections. Restricted use of antibiotics is therefore important since it contributes not only to the reduction of bacterial resistance, but also towards reduction of cardiovascular risks. This confirms the compelling need to use antibiotics in an indication-oriented and targeted manner for bacterial infections. The restrictive use of antibiotics is therefore not only important to reduce bacterial resistance but also contributes to the reduction of cardiovascular risks.

\section{REFERENCES}

1. Fleming-Dutra KE, Hersh AL, Shapiro DJ, Bartoces $\mathrm{M}$, Enns EA, File TM Jr, et al. Prevalence of inappropriate antibiotic prescriptions among US ambulatory care visits, 2010-2011. JAMA 2016; 315: 1864-73.

2. Linder JA, Brown T, Lee JY, Chua K-P, Fischer MA. 1632. Non-Visit-Based and Non-Infection-Related Ambulatory Antibiotic Prescribing. Open Forum Infect Dis. 2018; 5 (Suppl 1): S43.
3. Heianza Y, Zheng Y, Ma W, Rimm EB, Albert CM, $\mathrm{Hu}$ FB, et al. Duration and life-stage of antibiotic use and risk of cardiovascular events in women. Eur Heart J. 2019; 40: 3838-3845.

4. Nachimuthu S, Assar MD, Schussler JM. Druginduced QT interval prolongation: mechanisms and clinical management. Ther Adv Drug Saf. 2012; 3: 24153 .

5. Li X, Wang M, Liu G, Ma J, Li C. Association of macrolides with overall mortality and cardiac death among patients with various infections: a metaanalysis. Eur J Intern Med. 2016; 28:32-7.

6. Wong AYS, Chan EW, Anand S, Worsley AJ, Wong ICK. Managing cardiovascular risk of macrolides: systematic review and meta-analysis. Drug Saf. 2017; 40: 663-77.

7. Bin Abdulhak AA, Khan AR, Garbati MA, Qazi AH, Erwin P, Kisra S, et al. Azithromycin and risk of cardiovascular death: a meta-analytic review of observational studies. Am J Ther. 2015; 22: e122-e129.

8. Ray WA, Murray KT, Hall K, Arbogast PG, Stein CM. Azithromycin and the risk of cardiovascular death. $\mathrm{N}$ Engl J Med. 2012; 366: 1881-90.

9. Schembri S, Williamson PA, Short PM, Singanayagam A, Akram A, Taylor J, et al. Cardiovascular events after clarithromycin use in lower respiratory tract infections: analysis of two prospective cohort studies. BMJ 2013; 346: fi235.

10. Svanström H, Pasternak B, Hviid A. Use of clarithromycin and roxithromycin and risk of cardiac death: cohort study. BMJ 2014; 349: g4930.

11. Khosropour CM, Capizzi JD, Schafer SD, Kent JB, Dombrowski JC, Golden MR. Lack of association between azithromycin and death from cardiovascular causes. N Engl J Med. 2014; 370: 1961-2.

12. Chou HW, Wang JL, Chang CH, Lai CL, Lai MS, Chan KA. Risks of cardiac arrhythmia and mortality among patients using new-generation macrolides, fluoroquinolones, and $\beta$-lactam $/ \beta$ - lactamase inhibitors: a Taiwanese nationwide study. Clin Infect Dis. 2015; 60: 566-77.

13. Mortensen EM, Halm EA, Pugh MJ, Copeland LA, Metersky M, Fine MJ, et al. Association of azithromycin with mortality and cardiovascular events among older patients hospitalized with pneumonia. JAMA 2014; 311: 2199-208.

14. Winkel P, Hilden J, Hansen JF, Kastrup J, Kolmos HJ, Kjøller E, et al.; CLARICOR trial group. Clarithromycin for stable coronary heart disease increases all-cause and cardiovascular mortality and cerebrovascular morbidity over 10 years in the 
CLARICOR randomised, blinded clinical trial. Int J Cardiol. 2015; 182: 459-65.

15. Wong AY, Root A, Douglas IJ, Chui CS, Chan EW, Ghebremichael-Weldeselassie Y, et al. Cardiovascular outcomes associated with use of clarithromycin: population based study. BMJ 2016; 352: h6926.

16. Inghammar M, Nibell O, Pasternak B, Melbye M, Svanström H, Hviid A. Long term risk of cardiovascular death with use of clarithromycin and roxithromycin: a nationwide cohort study. Am J Epidemiol. 2018; 187: 777-85.

17. Gandhi S, Fleet JL, Bailey DG, McArthur E, Wald R, Rehman F, Garg AX. Calcium-channel blocker clarithromycin drug interactions and acute kidney injury. JAMA 2013; 310: 2544-53.

18. Fralick M, Macdonald EM, Gomes T, Antoniou T, Hollands S, Mamdani MM, Juurlink DN; Canadian Drug Safety and Effectiveness Research Network. Cotrimoxazole and sudden death in patients receiving inhibitors of renin-angiotensin system: population based study. BMJ 2014; 349: g6196.

19. Jie Z, Xia H, Zhong SL, Feng Q, Li S, Liang S, et al. The gut microbiome in atherosclerotic cardiovascular disease. Nat Commun. 2017;8:845.

20. Kita E, Sawaki M, Mikasa K, Oku D, Hamada K, Maeda K, et al. Proliferation of erythromycin- stimulated mouse peritoneal macrophages in the absence of exogenous growth factors. Nat Immun. 1993; 12: 326-38.

21. Xu G, Fujita J, Negayama K, Yuube K, Hojo S, Yamaji Y, et al. Effect of macrolide antibiotics on macrophage functions. Microbiol Immunol. 1996; 40: 473-9.

22. Robbins CS, Hilgendorf I, Weber GF, Theurl I, Iwamoto Y, Figueiredo JL, et al. Local proliferation dominates lesional macrophage accumulation in atherosclerosis. Nat Med. 2013; 19: 1166-72.

23. Kappel B, De Angelis L, Nonnast A, Stoehr R, Menghini R, Marx N, Federici M. Oral antibiotics increase atherosclerosis independently of diet. Eur Heart J. 2017; 38 (Suppl 1): 138.

24. Wang Z, Klipfell E, Bennett BJ, Koeth R, Levison BS, Dugar B, et al. Gut flora metabolism of phosphatidylcholine promotes cardiovascular disease. Nature 2011;472:57-63.

25. Scott NA, Andrusaite A, Andersen P, Lawson M, Alcon-Giner C, Leclaire C, et al. Antibiotics induce sustained dysregulation of intestinal $\mathrm{T}$ cell immunity by perturbing macrophage homeostasis. Sci Transl Med. 2018; $10 \quad(464)$ : eaao4755. https://doi.org/10.1126/scitranslmed.aao4755 